\title{
PSA Based Biomarkers, Imagistic Techniques and Combined Tests for a Better Diagnostic of Localized Prostate Cancer
}

\author{
Vlad Cristian Munteanu ${ }^{1,2, *}$, Raluca Andrada Munteanu ${ }^{3}{ }^{(0)}$, Diana Gulei ${ }^{3}{ }^{(0)}$, \\ Vlad Horia Schitcu ${ }^{1}$, Bogdan Petrut ${ }^{1,2}$, Ioana Berindan Neagoe ${ }^{4,5}$, \\ Patriciu Achimas Cadariu ${ }^{6,7}$ and Ioan Coman ${ }^{2,8, *}$ \\ 1 Department of Urology, The Oncology Institute "Prof Dr. Ion Chiricuta", 400015 Cluj-Napoca, Romania; \\ schitcu@yahoo.com (V.H.S.); bogdan.petrut@gmail.com (B.P.) \\ 2 Department of Urology, "Iuliu Hatieganu" University of Medicine and Pharmacy, \\ 400012 Cluj-Napoca, Romania \\ 3 MedFuture-Research Center for Advanced Medicine, "Iuliu Hatieganu" University of Medicine and \\ Pharmacy, 400337 Cluj-Napoca, Romania; muresan.raluca.andrada@gmail.com (R.A.M.); \\ diana.c.gulei@gmail.com (D.G.) \\ 4 Research Center for Functional Genomics, Biomedicine and Translational Medicine, "Iuliu Hatieganu” \\ University of Medicine and Pharmacy, 400337 Cluj-Napoca, Romania; ioananeagoe29@gmail.com \\ 5 Department of Functional Genomics and Experimental Pathology, The Oncology Institute \\ "Prof. Dr. Ion Chiricuta", 400015 Cluj-Napoca, Romania \\ 6 Surgery Department, The Oncology Institute "Prof. Dr. Ion Chiricuţă", 400015 Cluj-Napoca, Romania; \\ Pachimas@umfcluj.ro \\ 7 Department of Surgery and Gynecological Oncology, the University of Medicine and Pharmacy \\ "Iuliu Hatieganu", 400337 Cluj-Napoca, Romania \\ 8 Department of Urology, Clinical Municipal Hospital, 400139 Cluj-Napoca, Romania \\ * Correspondence: vladcristian.munteanu@gmail.com (V.C.M.); jcoman@yahoo.com (I.C.)
}

Received: 3 August 2020; Accepted: 8 October 2020; Published: 10 October 2020

\begin{abstract}
Prostate cancer represents the most encountered urinary malignancy in males over 50 years old, and the second most diagnosed after lung cancer globally. Digital rectal examination and prostatic specific antigen were the long-time standard tools for diagnosis but with a significant risk of overdiagnosis and overtreatment. Magnetic resonance imaging recently entered the diagnosis process, but to this date, there is no specific biomarker that accurately indicates whether to proceed with the prostate biopsy. Research in this area has gone towards this direction, and recently, serum, urine, imagistic, tissue biomarkers, and Risk Calculators promise to help better diagnose and stratify prostate cancer. In order to eliminate the comorbidities that appear along with the diagnosis and treatment of this disease, there is a constant need to implement new diagnostic strategies. Important uro-oncology associations recommend the use of novel biomarkers in the grey area of prostate cancer, to better distinguish the next step in the diagnostic process. Although it is not that simple, they should be integrated according to the clinical policies, and it should be considered that statistical significance does not always equal clinical significance. In this review, we analyzed the contribution of prostate-specific antigen (PSA)-based biomarkers (PHI, PHID, 4Kscore, STHLM3), imagistic techniques (mp-MRI and $\mathrm{mp}$-US), and combined tests in the early diagnosis process of localized prostate cancer.
\end{abstract}

Keywords: prostate cancer; biomarkers; screening; prostate-specific antigen; magnetic resonance imaging; prostate health index; 4Kscore; Stockholm 3 test; multiparametric ultrasound 


\section{Introduction}

Biomarkers indicate the presence of a disease, and ideally, they should be identified through non-invasive and inexpensive tests. Specificity and sensitivity are important in order to accurately differentiate malign from the benign process and distinguish between indolent and aggressive tumors. In consequence, the use of these tests should help physicians confirm cancers, diagnose them early, evaluate the risk of progression or recurrence, and could also monitor the effectiveness of treatment [1].

Prostate cancer (PCa) is the second most common cancer among men, and globally, it represents $13.5 \%$ of all cancers, and it occupies the fifth place in terms of mortality [2,3]. In 2018, 1.3 million new patients received the diagnosis of prostate cancer, and 359,000 died from this disease [2,4]. It is age-specific, men with ages above $45-50$ years being at risk, but it also presents a genetic predisposition, for example, African and Caribbean ethnic groups being at high risk compared to other ethnic groups [5].

Even though direct rectal examination (DRE) was the first method of screening PCa, there is still much debate about its usefulness. As reflected in the European Association of Urology (EAU) Guidelines in 2020, the sensitivity and specificity of DRE in the primary care settings is below $60 \%$ and is not recommended for the exclusion of PCa [6].

The Food and Drug Administration (FDA) first approved the usage of prostate-specific antigen (PSA) in PCa in 1986, and this revolutionized the diagnosis process of $\mathrm{PCa}$, and allowed the detection in earlier stages, in contrast to previously used techniques where most of the cases were diagnosed in locally advanced or metastases stages. This tool helped physicians improve prostate cancer screening and consequently reduce the PCa specific mortality [7].

At this moment, systematic biopsy remains the gold standard in PCa diagnosis, as stated by EAU Guidelines from 2020 with a $1 \mathrm{~b}$ level of evidence (evidence obtained from at least one randomized trial) [8].

Once the diagnosis has been established, some patients have the option for active surveillance [9] while others, with more aggressive cancers, need active treatment (radical prostatectomy or radiation therapy) [10], both with short- and long-term complications and changes in quality of life. The perioperative complications at 30 days were $21 \%$ for the patients that underwent radical prostatectomy; the long-term complications were urinary incontinence in $17 \%$ of cases, erectile dysfunction in $81 \%$ of cases, and bowel dysfunction in $12 \%$ of cases [11]. Up to $47 \%$ of men presented a degree of incontinence at 6 months after surgery, which downgraded to $18 \%$ at 6 years, erectile function decreased by $50 \%$ for potent men at 6 months after treatment and did not recover over time [12]. Of the patients, $65 \%$ presented severe erectile dysfunction, and digestive complications (constipation, diarrhea, appetite loss), cognitive, emotional, and functional scores were worse for treated patients compared to controls. [13].

Diagnosing and treating based only on PSA can lead to overdiagnosis and overtreatment of tumors that are not clinically significant $[14,15]$. Along with the comorbidities that come with those, many researchers started to focus on new biomarkers for diagnosing and staging PCa, evaluating them from the blood (PHI, 4Kscore, S3M), from urine (PCA3, TMPRSS2-ERG, TDRD1, DLX1, HOX6), or through radiographic modalities (multiparametric magnetic resonance imaging(mpMRI), multiparametric ultrasound (mpUS) [14,15]. Risk calculators have been developed, they combine age, PSA, DRE, trans-rectal ultrasound (TRUS), PSA density (PSAD), prostate volume, and more recent MRIs to better differentiate indolent cancers from clinically significant ones, but they can be improved [16,17].

In this review, we aim to discuss the positive value brought by PSA-derived biomarkers, MRI, US, and the combinations of these methods for a better early diagnosis process of localized PCa, with a PSA level under $10 \mathrm{ng} / \mathrm{mL}$. We will explain how to interpret all these biomarkers and how they could be applied in day-to-day clinical practice in the screening and diagnosis process, in the active surveillance process, and in scenarios with previous negative biopsies. These tools combined should offer a better risk stratification of PCa. The chosen assays and imaging techniques are non-invasive tests with 
high sensitivity and specificity for diagnosing PCa, they can help discriminate between benign and aggressive cancers.

\section{Classic PCa Diagnosis Pathways}

\subsection{DRE (Digital Rectal Examination)}

DRE is not recommended routinely as a tool in PCa primary care, which is a factor that could contribute to unnecessary biopsies, being a consequence that leads to overdiagnosis and overtreatment [18]. Despite this aspect, it is suggested that DRE has not lost its value completely. For example, patients with PSA levels under $4 \mathrm{ng} / \mathrm{mL}$ DRE had a sensitivity rate of $35 \%$. In these cases, DRE represents a tool that should be used in the clinical evaluation of men with PCa under Active Surveillance (AS) regardless of PSA values, even though DRE alone cannot rule out the presence of clinically significant $\mathrm{PCa}$ (csPCa). Men under AS with an initial DRE negative, which becomes positive overtime, should be closely monitored [19].

Abnormal DRE is not universally defined, it is a subjective maneuver, and it does not present any technical aspects. For example, in a Canadian survey, half of the graduating students, during their clerkship training, had never performed DRE, only half of the primary care physicians felt confident in detecting nodules through DRE [18].

DREs could help in deciding whether to rebiopsy patients on screening programs. If DRE is normal, the rebiopsy could be postponed until a later screening meeting; this can reduce the risk of diagnosing indolent cancers and can reduce the number of unnecessary biopsies [20].

In the ERSPC (European Randomized Study of Screening for Prostate Cancer) trial, 15\% of DREs results were considered false positive because they could not be confirmed on biopsy [21].

\subsection{PSA (Prostate-Specific Antigen) and fPSA (freePSA)}

Secreted by the prostatic epithelium, PSA is organ-specific, not cancer-specific, which means that other pathologies also influence PSA levels, such as prostatitis or benign prostatic hyperplasia $(\mathrm{BPH})$, androgen levels, DRE, body mass index (BMI) (hemodilution of PSA), prostatic trauma (biopsy), urinary retention, ejaculation under $24 \mathrm{~h}$ [7].

A PSA level above $4 \mathrm{ng} / \mathrm{mL}$ is considered suspect for PCa, although levels between $4-10 \mathrm{ng} / \mathrm{mL}$ are considered to be in a grey zone. Due to this particularity concerning PSA, overdiagnosis and overtreatment are risks, with severe and unnecessary complications [1]. Evidence shows that up to $25 \%$ of patients with normal PSA values can have underlying PCa [5].

All these data show that PSA alone is not a good predictor of biopsy results, and many studies suggest that it should be accompanied by other biomarkers to improve the outcome $[22,23]$.

Some authors are recommending precaution when using PSA screening for PCa [24]. The argument states that it is more likely that patients will suffer because of the biopsy or radical treatment complications. Screening data from major randomized controlled trials like The Cluster Randomized Trial of PSA Testing for Prostate Cancer (CAP), The European Randomised Study of Screening for Prostate Cancer (ERSPC), The Prostate, Lung, Colorectal and Ovarian (PLCO), with a screening group and a control group, over 10 years, showed that for every 1000 people, two patients from the screening group will die of PCa. Three patients from the non-screening group will have the same outcome. From the first cohort, 94 patients presented blood in semen, 45 experienced pain, 19 fever, 67 blood in urine, and one sepsis, all being complications of prostate biopsy. Moreover, from the treated group, 25 patients had erectile dysfunction, without the possibility of penetration, and three presented urinary incontinence due to radical treatment. In the screening group, PSA screening helps diagnose more cancers at any stage (18 more/1000 patients) than in the non-screening group and identifies more localized cancers (14 more/1000 patients). This small benefit brought by PSA can be associated with short or long-term complications [24]. 
For men who underwent prostate biopsies for risk of $\mathrm{PCa}$, the 30 days complications rates were $17 \%$, of which $8.3 \%$ were non-sepsis genito-urinary infections, $7.3 \%$ with bleeding complications, and $2.9 \%$ presented urinary retention. Previous use of fluoroquinolones and anticoagulants, age above 70, previous cancer diagnoses were associated with a higher risk of complications sepsis or hospitalization [25]. Other authors report a $1.5 \%$ risk of infection, $12.5 \%$ hematuria, $3.6 \%$ hematospermia, and $7 \%$ pain after prostate biopsy [26]. When comparing the complication rates between transrectal (TR) and transperineal (TP) prostate biopsies, the urosepsis rate was $2.46 \%$ vs. $0.4 \%$, urinary retention $2.76 \%$ vs. $3.63 \%$, hematuria $0.23 \%$ vs. $0.81 \%$ [27].

Schröder FH et al., 2014 determined that long-term screening for PCa can reduce PCa mortality by about 9\% [25]. To prevent one death from PCa, 1410 men needed to be screened, and 48 of them had to be treated [21]. In the same trial, 10.4\% of PSAs resulted in false-positive results on biopsy, but other studies report up to $75 \%$ [21].

Even though it is not recommended to only use the PSA value for PCa diagnosis, to date, it is the most useful tool for follow-up after active treatment [1]. It will continue to be in the spotlight for prostate cancer because it has implications in angiogenesis, invasion, metastasis, and cancer signaling [28].

A large percentage of PSA is bound to $\alpha 1$-antichymotrypsin, $\alpha 2$-macroglobulin, and $\alpha 1$-proteinase inhibitor, in a proportion of about $85 \%$, the rest is represented as freePSA (fPSA) [1]. The freePSA ratio $(\mathrm{fPSA} \%)$ is $\mathrm{fPSA} / \mathrm{tPSA} \times 100$. Usually, a high PSA and a low $\mathrm{fPSA} \%$ is associated with more aggressive PCas. FreePSA is also used as a component of PHI score and 4Kscore [1].

A computer model was created based on the data of the PLCO trial, the area under the curve (AUC) was calculated for the PCa diagnostic prediction. PSA showed an AUC of 0.63, freePSA an AUC of 0.50, free to total ratio an AUC 0.65, and the computer model an AUC of 0.72. FreePSA alone is a poor diagnosis tool, but integrated into other tests (PHI, 4Kscore), it raises their accuracy [29].

In Table 1, the prostate cancer screening indications offered by AUA, EAU, ESMO, ACS based on PSA are highlighted.

Table 1. Prostate-specific antigen (PSA)-based screening recommendations.

\begin{tabular}{|c|c|}
\hline $\begin{array}{c}\text { Screening Recommendation } \\
\text { Associations }\end{array}$ & Recommendations \\
\hline $\begin{array}{c}\text { AUA (American Association of } \\
\text { Urology) [30] }\end{array}$ & $\begin{array}{l}\text { PSA screening for men } 55-70 \text { years old } \\
\text { Urinary, serum biomarkers, imaging, Risk Calculators can be used for men with a } \\
\text { suspicious PSA level } \\
\text { Screening at } 2 \text { years or more can be applied to reduce the harm of screening } \\
\text { No screening if life expectancy is }<15 \text { years old, or men }>70 \text { years old }\end{array}$ \\
\hline $\begin{array}{c}\text { EAU (European Association of } \\
\text { Urology) [8] }\end{array}$ & $\begin{array}{l}\text { PSA screening for men over } 50 \text {-year-old or over } 45 \text { if they had a family history of PCa, } \\
\text { African descent or over } 40 \text { if carrying BRCA2 mutations } \\
\text { Men with PSA level }>1 \mathrm{ng} / \mathrm{mL} \text { at } 40 \text {-year-old or }>2 \mathrm{ng} / \mathrm{mL} \text { at } 60 \text {-year-old are at risk } \\
\text { Men with PSA } 2-10 \mathrm{ng} / \mathrm{mL} \text { and normal DRE, prior to biopsy, use additional tests (PCA3, } \\
\text { PHI, } 4 \text { Kscore, Kallikreins, TMPRSS2-ERG or Risk Calculators, Imaging (mpMRI) } \\
\text { PSA screening every } 2 \text { years for those at risk }\end{array}$ \\
\hline $\begin{array}{l}\text { ESMO (European Society of } \\
\text { Medical Oncology) [31] }\end{array}$ & $\begin{array}{l}\text { Subclinical PCa is common in men }>50 \text {-year-old } \\
\text { PSA screening for men } 55-70 \text { years old } \\
\text { PSA level }>1 \mathrm{ng} / \mathrm{mL} \text { for men at } 40 \text { years old and }>2 \mathrm{ng} / \mathrm{mL} \text { for men at } 60 \text {-year-old } \\
\text { represents a risk } \\
\text { Early PSA screening for men }>50 \text { years old, }>45 \text { with a family history of PCa, } \\
\text { African-American, and BRCA } 1 / 2 \text { carriers } \\
\text { Do not test if life expectancy is }<10 \text { years } \\
\text { Use Risk Calculators or mpMRI before biopsy }\end{array}$ \\
\hline $\begin{array}{c}\text { ACS (American Cancer Society) } \\
{[32]}\end{array}$ & $\begin{array}{l}\text { Informed PSA screening for men }>50 \text { years old, }>45 \text { if African-American or men with } \\
\text { first-degree relative diagnosed with PCa (under the age 65), }>40 \text { years old if they have } \\
\text { more than one first degree relative with PCa } \\
\text { Early screening for men with PSA level } 2.5 \mathrm{ng} / \mathrm{mL} \\
\text { If the biopsy is negative, additional tests can help (PHI, } 4 \text { Kscore, PCa3, ConfirmMDx) }\end{array}$ \\
\hline
\end{tabular}




\subsection{PSA Density (PSAD)}

PSAD is defined as the PSA value divided by the volume of the prostate, with thresholds between $0.08 \mathrm{ng} / \mathrm{mL} / \mathrm{cc}$ and $0.15 \mathrm{ng} / \mathrm{mL} / \mathrm{cc}$, it can be helpful in making biopsy decisions, when the PSA $<10 \mathrm{ng} / \mathrm{mL}$. It was compared with PSA in terms of diagnosing $\mathrm{PCa}$, and proved to be significantly superior $(p=0.011)[33,34]$. Combined with PSA, fPSA, or even with mpMRI, it can help better discriminate whether to proceed with the prostate biopsy [35]. Adding PSAD in this landscape can lead to better discrimination of PCa than PSA alone (AUC 0.75 vs. $0.73, p<0.05$ ) [36].

\subsection{MpMRI (Multiparametric Magnetic Resonance imaging)}

Pre-biopsy MRI is now part of the EAU guidelines for biopsy naive patients [8] even though some authors advise against it, contesting the idea that systematic TRUS biopsy would miss many high-grade PCas. According to Kasivisvanathan et al., in the PRECISION study in 2018, MRI increased the diagnosis of Gleason grade (GG) 5 PCa by $2.8 \%$. The contesting authors interpreted this as about 10,000 cases missed by TRUS per year, leading to an increased number of metastatic cancers, but that is not the present case [37]. Pre-biopsy MRI with targeted biopsies does increase the risk of over-diagnosing. Still, it should also be interpreted according to tumor size, coefficient of diffusion, PSA density, and systematic biopsy results [37].

\section{Non-Invasive Biomarkers in PCa Diagnosis}

Below, we will detail the advantages of these biomarkers over PSA in the screening and diagnosis process, in monitoring patients on AS, in scenarios with previous negative biopsies, as well as their power to differentiate between indolent and aggressive tumors. The biomarkers detailed below were chosen because they all contain the PSA component and PSA isoforms, and they can be easily implemented in clinical practice.

\subsection{Prostate Health Index (PHI) and Prostate Health Index Density (PHID)}

PHI is a blood-based diagnostic test that combines PSA, freePSA, and [-2]pro-PSA (precursor of PSA) values. The test was validated, especially for diagnosing clinically significant cancers for patients with a PSA of $4-10 \mathrm{ng} / \mathrm{mL}$. The utility of the test is that it can help reduce unnecessary biopsies, and it proved higher specificity and sensitivity than total PSA (tPSA) and free PSA \% [1], with cutoff values that can range between $20-40$ [38,39]. PHID is defined as PHI divided by prostate volume with a cutoff above 0.9 [34].

Eveline A M Heijnsdijk et al. in 2016 created a microsimulation based on the ERSPC trial results, which evaluated the effects of PHI added to the PSA screening. They found that using PHI could have reduced the number of negative biopsies by $23 \%$ at a PSA level $3-10 \mathrm{ng} / \mathrm{mL}$, but using a cutoff value of $35 \%$, it can decrease the biopsies number by $42 \%$ [40].

Taken together, PHI and PHID proved to be better than PSA, fPSA \% and PSAD when deciding which patients should undergo biopsy and which should not. For all cancers diagnosed, the AUC was 0.7222 and 0.739 for PHI and PHID, but the AUCs for PSA, PPSA\%, and PSAD were 0.595, 0.612, and 0.698 . When focusing on clinically significant cancers, the receiver operating characteristics (ROC) for PHI and PHID were 0.757 and 0.764 , but for PSA, fPSA \% and PSAD, they were only $0.635,0.627$, and 0.732. For all cancers and significant cancers, the differences between PHI, PHID vs. PSA, fPSA $\%$, were statistically significant, but not statistically significant when PHI and PHID were compared with PSAD. With a cutoff value of $>40$ for PHI and $>0.9$ for PHID, these two tests excluded the need for biopsy for $20 \%$ of patients and only missed 1 Gleason $8 \mathrm{PCa}$, which after prostatectomy downgraded to Gleason 7(4+3), pT2a [34].

In the multicentric study, PRIM (PHI to refIne MRI), conducted by Lois Kim et al. 2020 on a cohort of 545 men, PHI proved better detection of significant $(G G \geq 2)$ PCa than PSA and PSAD with an AUC of 0.82 vs. $0.70,0.79$. When mpMRI was negative, PHI showed an AUC of 0.78 vs. 0.64 and 
0.76 (PSA and PSAD) in detecting PCa GG $\geq 2$. For PHI $\geq 20$, the negative predictive value (NPV) was 0.85 with $1.1 \%$ missed cancers, and for PHI $\geq 30$ the NPV was 0.9 with $7.7 \%$ missed cancers. When using the PHI $\geq 30$, and considering GG $\geq 2$ as an endpoint, PHI managed to reduce the number of mpMRIs by $35 \%$ with $9 \%$ missed cancers and reduced the unnecessary biopsies by $40 \%$ with $8 \%$ missed tumors [41].

Men on active surveillance can benefit from PHI and PHID, which can help reclassify the tumor grade. Patients with higher PHI values had a higher chance of reclassification. A PHI score under 25.6 and a Prostate Imaging-Reporting and Data System (PI-RADS)v2 lesions $\leq 3$, could help avoid $20 \%$ of prostate biopsies while missing only $2.6 \%$ that would have been reclassified, but if MRI examination is negative, as many as $41 \%$ of biopsies could be bypassed while missing $11 \%$ of tumors that would have been reclassified [42].

Men with PCa can be identified and separated from healthy men using PHI (AUC 0.887). Still, for differentiating prostate cancer patients from patients with $\mathrm{BPH}$, p2PSA showed better results than PHI (AUC 0.7333 vs. 0.639). As the tumor becomes more aggressive and the disease is more advanced, the level of PHI increases, and if there are positive margins, it remains high even after radical prostatectomy. PHI proved to be an independent predictor for diagnosis and prognosis [43].

PHI is a PSA-based blood marker that showed its usefulness in biopsy naïve patients, saving a lot of them from unnecessary biopsies and possible biopsy complications. It can differentiate PCa patients from healthy ones and patients with BPH. If the mpMRI is negative, it helps urologists decide whether to biopsy or not and if it is performed before the MRI test, it can also help avoid it, a decision which also has economic implications. Active surveillance patients can also benefit from the PHI test in the decision to undergo rebiopsy or not. With all the benefits that it has shown, large randomized studies are needed to establish its definitive role.

\subsection{Kscore (4 Kallikreins Score)}

The 4 kscore (4K) is associated with the test results of four kallikreins: totalPSA, freePSA, intactPSA (isoform of freePSA), and human kallikrein 2 (hk2). The results correspond to one of the three risk categories: low risk at $1-7.5 \%$, moderate risk at $7.5-19 \%$, and above $20 \%$ is considered high risk for the positive disease at biopsy. It can be used for patients with previous negative biopsy or patients with an indication for prostate biopsy. At a PSA $>3 \mathrm{ng} / \mathrm{mL}$, the 4 Kscore is more sensitive for high-grade cancer detection than clinical variables alone. For PHI and 4 Kscore, the diagnosis performance was similar, but they each outperformed PSA age stratification for the high-grade cancer prediction [1].

For a cohort of 1012 Caucasian men undergoing a prostate biopsy, a 4Kscore spares 252 biopsies, while missing 19 of 195 aggressive PCa detected on biopsy. In the same study, the 4Kscore showed better results than PSA for reclassifying specimens at first biopsy (AUC 0.78 vs. 0.74), but not at subsequent ones (AUC 0.75 vs. 0.76) [44].

Using serum samples from the ERSPC clinical trial, the Rotterdam section, Andrew J. Vickers et al. 2010 calculated the 4 Kscore, and found that it reduces the number of biopsies by $36 \%$, postponing the diagnosis of 43 low-grade PCa and 4 high-grade PCa from a total of 1000 biopsies. The base model (DRE, PSA, and age) versus the full model (age, PSA, freePSA, and hk2) showed an AUC of 0.585 versus 0.711 for diagnosing any cancer, and AUC 0.709 vs. 0.798 in detecting high-grade cancer. Biopsy based on the kallikrein model leads to superior clinical outcomes. In this setting, adding PSA velocity did not improve the biopsy outcome [23].

In the same clinical trial, but at rebiopsy (the first biopsy was negative), the AUC for 4Kscore vs. base model (PSA, age, DRE) was 0.681 vs. 0.584 for detection of any PCa, and 0.873 vs. 0.764 in detecting high-grade cancer. In this context, the authors believe that by applying the 4 Kscore, it is possible to reduce biopsies by $82 \%$ (risk cancer of $20 \%$ ) while postponing the diagnosis for 64 low-grade PCa and 3 high-grade PCa (none Gleason $\geq 8$ ) per 1000 patients [22].

At Skane University Hospital, between 2004 and 2010, a screening that included 749 men with PSA levels $\geq 3.0 \mathrm{ng} / \mathrm{mL}$, fPSA $\% \leq 20 \%$, or abnormal DRE underwent biopsy. The authors created a $4 \mathrm{~K}$ 
model with higher discrimination than the classic model that included PSA, age, and DRE. It better predicted high-risk PCa, with an AUC of 0.777 for the 4Kscore, and only AUC: 0.719 for PSA and age. When the model was adjusted for age, $4 \mathrm{~K}$, and DRE, the AUC raised to 0.784 . This model could reduce unnecessary biopsies by about $25 \%$, and miss about $6 \%$ of high-grade cancers [45].

In a multiethnic group (African Americans, Japanese, Latinos, Native Hawaiians, and Whites) divided into controls and disease subsets, the 4Kscore was compared with PSA+fPSA and PSA alone. It showed an AUC of 0.748 vs. 0.7111 and 0.669 for any given PCa. When evaluating these tests for the accuracy of finding aggressive PCa, the AUC results were 0.782 vs. 0.739 and 0.685 . This prospective study showed the 4Kscore's accuracy to discriminate benign from malignant cases and that it can be superior to PSA and fPSA in diagnosing aggressive and non-aggressive tumors [46].

The Rotterdam Prostate Cancer Risk Calculator (RPCRC) was compared with the 4Kscore, and they both showed similar AUCs (0.88 vs. 0.87), but combined, they improved the AUC to 0.89, which is statistically better than each test alone. The tests used either independently or synergistically could reduce the number of unnecessary biopsies by $65-66 \%$ while missing $14-16 \%$ of csPCas [47].

The 4 Kscore showed superiority over PSA, fPSA, PSA+fPSA, DRE in terms of diagnosis indolent and aggressive PCa, and specimen reclassification. It can be used in cases of high suspicion PCa with previous negative biopsies, and it also showed superiority over a Risk Calculator. Still, the two together had the best outcome in detecting PCas.

\subsection{The Stockholm-3 Model for Prostate Cancer Detection (STHLM3)}

The STHLM3 model or S3M model consists of a combination of blood biomarkers (free PSA, PSA, intact PSA, MSMB (microseminoprotein beta), MIC1 (macrophage inhibitory cytokine-1), hk2), genetic polymorphisms (232 SNPs) and clinical details such as age, previous biopsies or prostate exams [48]. This model proved to be more sensitive in diagnosing PCa than PSA for men between the age of 50-70 [14,49]. It can serve as a biomarker for high-risk cohorts such as African Americans (AUC 0.852), and Hispanic Caucasians (AUC 0.895) [48].

Between 2012 and 2015, 59,149 participants with ages between 50-69 underwent PSA and S3M testing in the STHML3 study. Patients with PSA levels above $3 \mathrm{ng} / \mathrm{mL}$ and S3M suggestive for Gleason 7 underwent biopsy (7416 men). The S3M test showed an AUC of 0.75 compared to PSA (AUC 0.58). The authors concluded that S3M could reduce unnecessary biopsies up to $34 \%$ without the risk of missing any GS $\geq 7$ tumors [50].

Performing MRI at an S3M risk above 10\% could reduce the number of MRIs and prostate biopsies by $38 \%$, and it could diagnose $42 \%$ less insignificant PCa and miss $8 \%$ of csPCa [51]. The S3M-MRI prediction model proved to be superior in predicting an International Society of Urological pathology (ISUP) grade $>2$ PCa than the S3M model alone or only MRI (AUC 0.88 vs. 0.86 vs. 0.83 ) [52].

This test vas validated on a cohort of 60,000 men, and it showed very good results in separating indolent from clinically significant tumors, and it showed superiority to PSA in the PCa diagnosing process. It is clinically available only in Denmark, Norway, Sweden and Finland but the downside is that it is available only in these countries.

\section{Imagistic Techniques}

MRI is already integrated into most guidelines concerning PCa diagnosis. This examination is recommended before the biopsy, in the case of abnormal PSA value or abnormal DRE [8]. The US is an essential tool that every urologist should know how to handle. It is low cost, non-invasive, and it can be used in a variety of actions, from a simple consultation to post-surgery follow-up. The technique advanced and, nowadays, it can be used in the diagnosis process of PCa.

\subsection{Mp-MRI (Multiparametric Magnetic Resonance Imaging)}

This can increase detection, especially for anterior lesion, and it can accurately measure the prostate volume, tumor volume, aggressiveness, invasion of the capsule, or neurovascular bundles. 
Mp-MRI combines T2 imaging, apparent diffusion coefficient (ADC) calculations, dynamic contrast enhancement (DCE), diffusion sequences, and sometimes spectral MRI. It presents high sensitivity for tumors above $1 \mathrm{~cm}^{3}$, Gleason grade above 1, but for smaller lesions, or lower grades, it is limited [53]. In contrast, bi-parametric MRI (bp-MRI) uses only T2 weighted image (T2W), diffusion-weighted imaging (DWI), and ADC, lowering the investigation time from $27 \mathrm{~min}$ for mp-MRI to just $17 \mathrm{~min}$ for bp-MRI, and being equally good in detecting PCa. Bp-MRI vs. mp-MRI presented a sensitivity of $0.94-0.96$ vs. $0.93-1.00$ and specificity of 0.15 vs. $0.04-0.16$ [53].

In the PRECISION study, Kasivisvanathan et al., in 2018, randomized 500 patients into a 1:1 (252 MRI TB (targeted biopsy) group and 248 into a systematic biopsy (SB) group. CsPCa was detected in 95 patients (38\%) from the first cohort and 64 patients $(26 \%)$ in the second one. Of positive MRIs, most lesions were categorized as PI-RADS $5(83 \%)$, followed by $4(60 \%)$ and $3(12 \%)$. The complications post-procedure were the same for both groups. MpMRI $\pm \mathrm{TB}$ proved to be superior to $\mathrm{SB}$, diagnosing less insignificant $\mathrm{PCa}$, and avoiding a quarter of unnecessary biopsies [54].

A recent study published by Andres Labra et al. in 2020 investigated a cohort of 122 patients with mpMRI suspicion of prostate cancer undergoing MRI-TRUS fusion biopsy and SB biopsy. The positive results for Gleason 6 score were $56 \%$ (MRI-US fusion) vs. $48 \%(\mathrm{SB})$, representing only a small difference $(p=0.049)$, but for Gleason score of 7 and above, the difference became larger, $65 \%$ vs. $46 \%(p<0.001)$. The fusion group scored better for patients with negative prior biopsies $(48.7 \%$ vs. $38.5 \%, p<0.13)$ and for patients with difficult lesions (anterior, transition, central zone) ( $68 \%$ vs. $27 \%, p<0.001)$. SB missed $20 \%$ of csPCA identified by MRI-TRUS biopsy. The mpMRI PI-RADS scores of $3,4,5$, identified cancer in $36.7 \%, 72.1 \%$ respectively in $90.3 \%$ of cases, in concordance with the pathological results. The authors state that if collecting only two cores from a targeted zone, one can omit $25 \%$ of csPCa [55].

MRI should be implemented for all patients considered for prostate biopsy. Bp-MRI is cheaper and faster than mp-MRI, and lacks complications related to contrast media. MRI-fusion biopsy seems to gain acceptance, but targeted biopsy alone is not the way, because in some cases, systematic biopsy identified 3.9\% more csPCa and 6.8\% PCa that were invisible to the MRI. In contrast, TB identified $12.1 \%$ PCa cases and $12.9 \%$ csPCA that SB omitted [56].

The NPV was $88 \%$ in detecting clinically significant PCa, which means that mp-MRI can overlook $12 \%$ of prostate cancers [57].

Another study published by Hashim U Ahmed et al. in 2020 included 576 men in a trial, that underwent mpMRI and afterward template prostate mapping (TPM) (with $5 \mathrm{~mm}$ distance sampling) biopsy and TRUS biopsy, each one blinded from the other. TPM detected $71 \%$ PCa, $40 \%$ csPCa, missed $2 \%$ (13 cases) csPCa diagnosed by TRUS. MpMRI showed $93 \%$ sensitivity, $41 \%$ specificity, NPV $89 \%$ and positive predictive value (PPV) 51\%. Of 158 (27\%) patients with negative MRI, 17 had csPCa(GS 3+4), detected at TPM biopsy. TRUS biopsy identified 452 insignificant PCa, of which 119 cases proved to be csPCa on TPM biopsy. If mpMRI is performed before the biopsy, about $25 \%$ of patients will avoid unnecessary biopsies, and it will improve the detection of csPCA and reduce the overdiagnosis and detection of clinically insignificant PCa. TRUS was performed after TPM, and this might contribute to the poor accuracy of TRUS. Since each test was blind, they could not evaluate the utility of MRI targeted prostate biopsies [58].

MpMRI did not prove an upgrade of PCa compared with SB in AS at two years follow-up in the ASIST trial, although MRI had a higher sensitivity for diagnosing csPCa (93\% vs. 48\%, p.0.0001). A lower upgrading rate suggests that MRI TB aimed at the lesion from the start and the $50 \%$ reduction in AS failure, reinforcing the role of MRI in AS management. The study was conducted before the introduction of PI-RADS v2 [58].

When mpMRI was compared with mpUS, on 82 patients with PSA, freePSA, density, and velocity above normal levels, mpMRI detected 54 lesions, of which 44 proved to be PCa (at biopsy), showing a sensitivity of $91 \%$ an specificity of $66 \%$, in contrast to mpUS, of which transrectal elastography (TRES) proved the best sensitivity (69\%), specificity $(44 \%)$, and contrast-enhanced ultrasound (CEUS) showed 
a sensitivity of only $40 \%$ but a specificity of $97 \%$. The authors concluded that the two investigations combined could help maximize the accuracy of prostate biopsy [59].

A systematic review conducted by Liang Zhen et al. in 2019 carried out between 2007 and 2017 included 29 studies of mpMRI, and found a pooled sensitivity of 0.87 and specificity of 0.68 . Comparing MRI 1.5T with 3T, it seems that the 3T has higher sensitivity. Biparametric MRI versus multiparametric MRI showed similar sensitivity and specificity with the cost of higher signal heterogeneity and higher risks of artifacts [60].

In patients with PSA levels above $10 \mathrm{ng} / \mathrm{mL}$ and positive DRE, mpMRI brings a negligible benefit for diagnosing PCa. In general, mpMRI could reduce the number of unnecessary biopsies by $25 \%$ and increase the identification of High Grade (HG) PCa by up to $28 \%$ [61].

When comparing mpMRI with Prostate Cancer Antigen 3 (PCA3) and PHI for rebiopsy, mpMRI proved a higher accuracy in diagnosing PCa [62].

In the same context, Marloes van der Leest et al. conducted a study in 2018, and performed a head to head comparison between MRI followed by MRI guided biopsy vs. MRI and TRUS biopsy, and found that if patients with only PI-RADS 3-5 lesions would undergo biopsy, about $49 \%$ of men would be spared, with $3 \%$ missed csPCas. All the patients in this study underwent transrectal ultrasound-guided biopsy (TRUSGB), but only patients with PI-RADS 3-5 lesions also underwent "in bore" magnetic resonance-guided biopsy (MRGB). A percent of $4 \%$ of clinically significant prostate cancers were MRI nonsuspicious and identified by TRUSGB. Using only the positive MRIs when deciding to undergo biopsy could diagnose less insignificant PCas (25\% TRUSGB versus 18\% MRGB), and lower the complications rates [63].

MpMRI has established its role in the diagnosis process of $\mathrm{PCa}$ in active surveillance, and it also showed superiority over other markers (PSA, fPSA, PHI, PCA3) [14,62]. Recent studies show that bPMRI has similar results but is faster and cheaper. The main disadvantages are represented by the costs and the learning curve. It is not a perfect tool, and we consider that the direction of research would be towards combined tests that can also lower the economic burden and fasten the diagnosis process until a definitive decision.

\subsection{Mp-US (Multiparametric Ultrasound)}

Traditional TRUS can be improved with the addition of Tissue Harmonic Imaging and spatial compound imaging. Micro-ultrasonography (MicroUS) is referred to as frequencies of $14-29 \mathrm{MHz}$, which can offer a spatial resolution of $50-70 \mu \mathrm{m}$, with an up to $94 \%$ increase in PCa detection rate and AUC of $0.60-0.80$. In this context, multicenter trials are necessary to confirm this new imaging device [64].

Strain elastography (SE) is a procedure where the prostate is compressed and decompressed with the transrectal transducer. It provides a color-coded image, with the less elastic zones colored in blue. It is not PCa specific, and the results for the moment are confounding [64].

In contrast to SE, shear-wave elastography (SWE), is another method of identifying stiff zones. Compression and decompression must be avoided; the prostate is color-coded, soft normal tissue with blue color and suspicious zones in red. In the case of false-negative MRI, SWE can find PCa in the peripheral zone, in $2 / 3$ cases. Two meta-analyses found that SWE had an $83-86 \%$ sensitivity and $85-89 \%$ specificity for detecting PCa, and AUC of 0.94. It can distinguish peripheral nodules from BPH or macro-calcifications, and it can estimate Gleason score from stiffness. SWE is not powerful enough to exclude PCa without a prostate biopsy [64].

Both SE and SWE present limitations when facing large prostate glands; not all stiff lesions are cancers and not all cancers present stiffness [64].

CEUS is a technique in which microbubbles are injected via the bloodstream and act as an ultrasound contrast agent. However, it does not enter the urinary collecting system or interstitial space, and it is not contraindicated in renal insufficiency or urinary obstruction and has a low risk of an anaphylactic reaction (0.014\%) [65]. It can identify vascular and microvascular architecture or 
irregularities around PCa. CEUS can enhance Doppler sensitivity from 54\% to 93\% and specificity from $79 \%$ to $87 \%$ in diagnosing hypervascular PCa nodules [64]. In a large prospective study, with 1024 patients, CEUS helped identify $20.5 \%$ more csPCa at biopsy, including $15.6 \%$ PCa missed by standard biopsy [65].

Mp-US is a concept derived from mpMRI, which includes improved B mode, vascular imaging, elastography, perfusion imaging, and volumetric imaging. It may include micro-Doppler elastography and CEUS. Its performance could be comparable to that of mpMRI [64].

The prostate risk identification-micro ultrasound (PRI-MUS) risk score represents a protocol that standardizes suspicious prostate lesion on TRUS with Micro-US (29 MHz). The Score ranges from 1-5, very low risk, some risk, intermediate-risk, significant risk, and very high risk. The authors agree that combining this protocol with multiparametric features can enhance the US power in diagnosing PCa, especially csPCa [66].

The initial experience at Cleveland Clinic with MicroUS, PRI MUS protocol, on 67 patients (38 PCa patients diagnosed), found that using the MicroUS after TRUS biopsy changed the diagnosis for eight (21\%) patients and found six (26\%) cases of csPCa that TRUS missed. In contrast, TRUS found seven PCa cases missed by MicroUS. A total of 19 patients underwent mpMRI targeted biopsy immediately after US biopsy (TRUS and MicroUS), and 10 out of those 19 patients were confirmed positive for PCa. Of all three techniques, MicroUS found two subjects positive for PCa where mpMRI and systematic biopsy were negative. In this study, the TRUS detection rate was $44 \%$. Still, adding the Micro-US it raised to $56.7 \%$ and brought a relative improvement of $26.7 \%$ increase in diagnosing PCa. There was no statistical difference of added value to the diagnosis between mpMRI and MicroUS [67].

If patients are unfit to undergo mp-MRI for different reasons (pacemaker, claustrophobia, etc.) [68], CEUS proves to be a safe and cost-efficient alternative for most patients. In a prospective study conducted by Zhu Yunkai et al. in 2018, on 1024 patients, 378 were histologically positive for PCa. CEUS-TB (targeted biopsy) provided 27 cases of ISUP upgrade compared with SB, and longer cancer cores. It diagnosed more significant PCa in 67 cases (28.7\% vs. $25.3 \%)$ and only ten more cases of insignificant PCa. In contrast, SB diagnosed 40 cases more insignificant than CEUS-TB, but it also found 32 cases of significant PCa missed by the other one. CEUS provided promising results for patients with PSA levels $\leq 10 \mathrm{ng} / \mathrm{mL}$, and prostate volumes of 30-60 mL [65].

Using a $7 \mathrm{MHz}$ probe, and color Doppler ultrasound (CDUS) and spectacular micro-vascular imaging (SMI), 74/119 (62.2\%) of patients were diagnosed with PCa (biopsy confirmed). Of these, SMI proved to be superior in identifying blood vessels, and it identified abnormal vascularity in $97.3 \%$ (72/74 patients) of cases vs. 90.5\% (67/74 patients) (CDUS). The abundance of vascularity demonstrated a positive correlation between SMI, CDUS, and Gleason score. SMI-TB detected 89.2\% (66/74) PCa cases vs. SB with 58.1\% (43/74) cases, SMI-TB also identified 21 PCa patients missed by SB, and five patients underdiagnosed by SB. In contrast, when diagnosing Gleason 6, SB proved superiority in $10.8 \%$ (8/74 patients) vs. $1.4 \%$ (1/74 patients) of cases [69].

In a study conducted by Giovanni Lughezzani et al., in 2018, 104 patients underwent mpMRI and then MicroUS. The ultrasound urologist was blinded to the MRI results. From a total of 104 patients, mpMRI identified 21 insignificant PCa cases, $35 \mathrm{csPCa}$, and 48 benign cases. At the same time, MicroUS suspected 83 patients from a total of 104, 15 of them were found to be insignificant PCa, 33 csPCa, and 35 benign cases. Of the 21 patients with no suspect lesions, 13 were benign, six insignificant PCa, and two csPCas. MicroUS showed a sensitivity of $94 \%$, specificity of $28 \%$ in finding csPCa. Of the $138 \mathrm{mpMRI}$ targeted lesions, 48 (33\%) were positive, 32 (23\%) were csPCa. MicroUS targeted 117 lesions, of which $39(33 \%)$ were positive. Of these, $33(28 \%)$ were csPCa. The two investigations were concordant in 61 (45\%) of 136 targeted lesions [70].

One of the advantages of ultrasound over MRI is that the investigation usually is faster, an US scan takes about 5 to $10 \mathrm{~min}$ and the MRI $25 \mathrm{~min}$. Besides that, the US has the advantage that it is portable [71]. 
A multicenter prospective study published in 2020, where uro-radiologist experts on mpMRI and expert urologists on ultrasound examinations with at least five years of experience each tried to compare the results of mpMRI and micro-US in detecting PCa. For identifying PCa GG $\geq 2$, micro-US had a sensitivity of $94 \%$ vs. mpMRI with $93 \%$, $(p<0.03)$ specificity of $22 \%$ vs. $23 \%$ ( $p=0.01$ for non-inferiority). Micro-US showed similar specificity and sensitivity compared with the mpMRI, and the authors stated that it represents a strong alternative, is easier to use, and is familiar to the urologic community [68].

MpUS represents a novel technique, unique features with a lot of interest by urologists. It has shown promising results; some studies even achieved similar sensitivities and specificities with mpMRI. It has the advantage of being a fast procedure, being easy to do, but being a novel technique and the learning curve represent disadvantages.

\section{Combined Tests}

From the biomarkers and techniques presented above, none of them are ideal, each one presents advantages and disadvantages. In the following chapter, we present studies that combined the above-mentioned tools in order to be more effective in the PCa diagnosis process.

Combining the 4Kscore and mp-MRI provides more clinical information and leads to fewer biopsies with similar clinical PCa diagnoses compared to each investigation alone. If the 4Kscore is of low risk $(<5 \%)$, it can even avoid MRI, or if it is of high risk $(>23)$, the patient can undergo biopsy without MRI. Mp-MRI was useful for intermediate-risk categories [57]. This association between tests allowed $51 \%$ (151 patients) of men to avoid unnecessary biopsies from a total of 300 who were evaluated. Among the 149 patients who underwent biopsies, 73 (49\%) had PCa, of whom 49 (33\%) had Gleason Score 7. The 4Kscore associated with mpMRI provided an AUC of 0.82 (0.75-0.89) in contrast to each test individually, 4Kscore AUC 0.70(0.62-0.79), mpMRI AUC $0.74(0.66-0.81) p=0.001$ [72].

The 4Kscore (PSA based test) and Select MDx (non-PSA based test) were combined to aid in deciding to perform the prostate biopsy or not, but the tests were discordant in $45.6 \%$ of patients. Compared to each other, the AUC was 0.830 for 4 Kscore and 0.672 for SelectMDx $(p=0.036)$ in detecting clinically significant PCa65 [73]. Select MDx is a gene panel that consists of HOXC6, DLX1, TDRD1 genes, and it showed an AUC of 0.86 for detecting csPCa. It can reduce unnecessary biopsies by $42 \%$ while missing only $2 \%$ of csPCa [15].

In order to better diagnose PCa, in 2019, Ugo Giovanni Falagario et al. proposed three strategies to combine 4 Kscore, mpMRI, and PSAD. The first strategy starts with the evaluation of the $4 \mathrm{~K}$ value. If it was $>7.5$ (intermediate or high risk), they conducted a mpMRI. If the mpMRI was positive, the patient underwent biopsy, but if it was negative, and 4Kscore was above 7.5, but under 18 (intermediate risk), the patient went under clinical follow-up. The second strategy was similar, but the mpMRI was conducted first. The third strategy was based on mpMRI, and if positive, then PSAD would be calculated (cutoff $<0.10 \mathrm{ng} / \mathrm{mL} / \mathrm{cm}^{3}$ ). The first and second strategy missed $2.7 \%$ of csPCa and the third only $1.3 \%$. In conclusion, 4 Kscore lowered the number of mpMRIs, without missing a lot of csPCa. In general, it is a fast and cheap test, but if added after mpMRI, it can raise the total costs [72].

Laura Wiemer et al. conducted a study published in 2020, where patients underwent mpMRI (PI-RADS V2); after that, they were subjected to blinded Micro-US (PRI-MUS), and finally standard biopsies (12 cores) were performed. An extra two cores were taken from each PCa suspect lesion visualized on MRI or MicroUS. The latter identified 17\% (27/159 patients) more PCas that had MRI negative targeted biopsies, and 20 of those patients were csPCa. From a total of 159 cases, in 58\% ( $46 \%$ were csPCa) the MRI showed the same results as MicroUS. In 26\% cases, MRI showed higher grading, and in $16 \%$ cases, micro-US showed higher grading, adding value in detecting csPCa. If standard biopsies had been eliminated, both methods would have missed only $3 \%$ of csPCa [74].

The classic diagnosis pathway includes PSA DRE and mpMRI, which leads straight to the prostate biopsy. Still, if the novel biomarkers would be performed before the biopsy, it would bet triage the patients that this invasive maneuver. The same thing is valid for previous negative biopsies or for 
patients under active surveillance. Figure 1 is a graphical representation of diagnosis and treatment strategies applied in the PCa investigation.

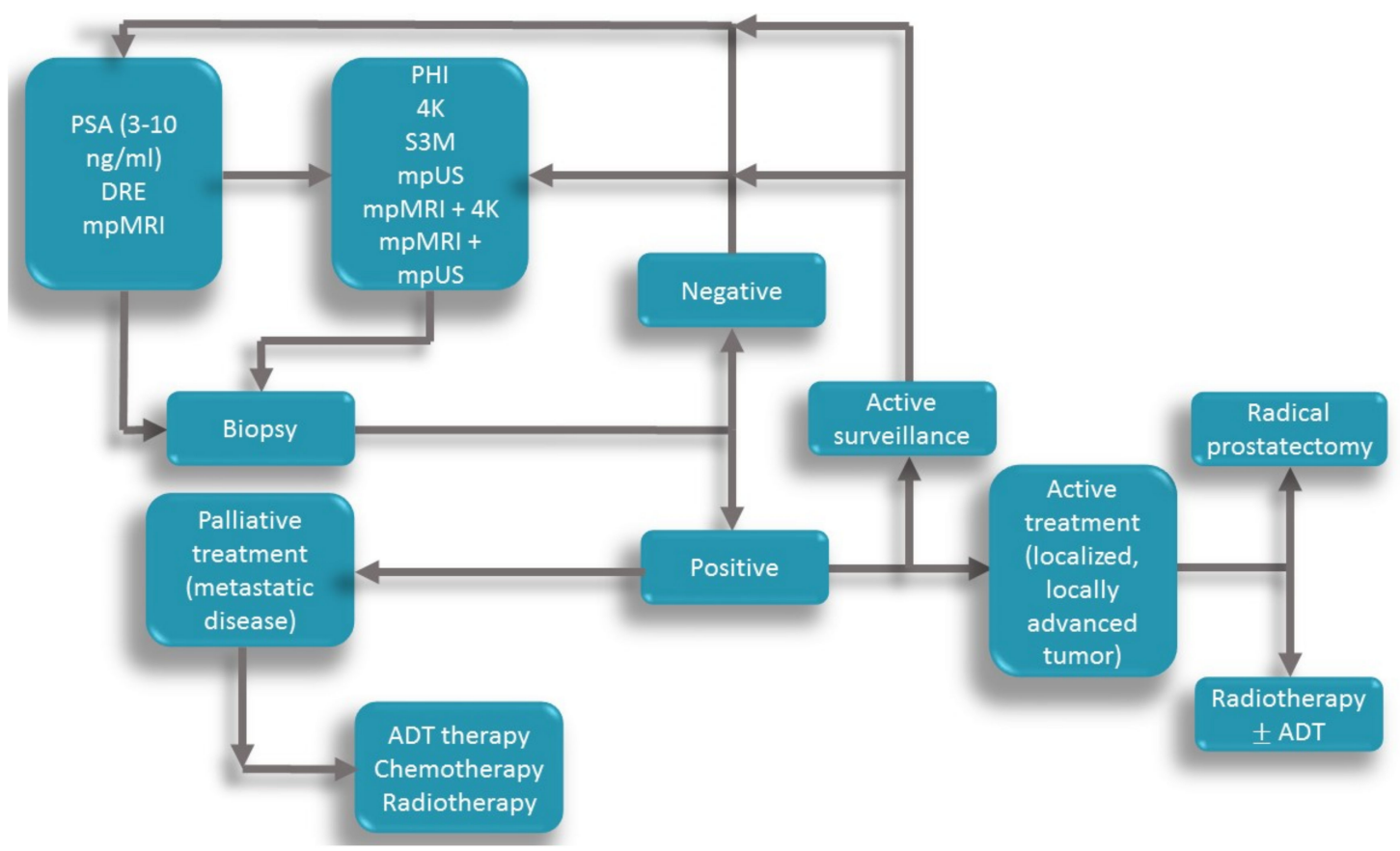

Figure 1. The pathway for diagnosis and treatment of prostate cancer. The diagram represents the patient's flow from the moment he presents a suspicious PSA level. First, direct rectal examination (DRE) and mpMRI are part of the standard of care. Before the biopsy, the patient should perform one of the tests that will further inform about the patient's risk of Pca and only then proceed with the biopsy. If it is negative, the patient returns to the diagnostic process, but if the biopsy is positive, the patient can enter active surveillance (for low-risk cancers) and undergo tests periodically, or if the cancer is intermediate- or high-risk, the patient will suffer curative treatment (radical prostatectomy of radiotherapy). If curative treatment is not an option, the patient (with metastatic disease) will undergo palliative care (ADT—androgen deprivation therapy, chemotherapy, radiotherapy).

\section{Other Perspectives}

The biomarkers that we consider of interest are described above and summarized in Table 2. Still, there are also other biomarkers to consider in the pre-diagnostic stage like PCA3, MiPS (from urine and blood), SelectMDX, and ExoDx prostate IntelliScore which are urine tests, or ConfirmMDx, which is a tissue test used in the case of negative biopsy and post-biopsy, and once the diagnosis is established, biomarkers like Oncotype Dx, ProMark, Prolaris, and Decipher have a prognostic role [75].

Table 2. Representative studies for recent advances in diagnose of PCa (inclusion criteria: study design and number of patients).

\begin{tabular}{ccccc}
\hline Marker/Technique & Number of Patients & Study Design & Cancer Detection & Other Details \\
PHI [41] & 545 & $\begin{array}{c}\text { Prospective, } \\
\text { multicentric study }\end{array}$ & $\begin{array}{c}\text { PHI AUC 0.82 } \\
\text { PSAD AUC 0.79 } \\
\text { PSA AUC 0.70 }\end{array}$ & $\begin{array}{l}\text { If MRI is negative, PHI AUC for positive PCa 0.78 } \\
\text { If PHI } \geq 30,35 \% \text { of MRIs could be avoided } \\
\text { Spared 40\% of biopsies } \\
\text { Missed } 8 \% \text { PCas }\end{array}$ \\
\hline 4Kscore [46] & 2224 PCa & $\begin{array}{l}\text { Prospective, } \\
\text { multicentric, } \\
\text { case-control, } \\
\text { multiethnic }\end{array}$ & $\begin{array}{c}\text { PSA+fPSA AUC 0.739 } \\
\text { PSA AUC 0.685 }\end{array}$ & $\begin{array}{l}\text { 4K AUC } 0.782 \\
\text { aggressive PCas } \\
\text { 4kscore can accurately differentiate between benign } \\
\text { and malign cases, indolent and aggressive tumors }\end{array}$ \\
\hline S3M [50] & 59149 & $\begin{array}{l}\text { Prospective, } \\
\text { population baased, } \\
\text { diagnostic trial }\end{array}$ & $\begin{array}{l}\text { S3M AUC 0.75 } \\
\text { PSA AUC 0.58 }\end{array}$ & 34\% of biopsies spared \\
\hline
\end{tabular}


Table 2. Cont.

\begin{tabular}{ccccl}
\hline Marker/Technique & Number of Patients & Study Design & Cancer Detection & \multicolumn{1}{c}{ Other Details } \\
\hline MRI [76] & 576 & $\begin{array}{c}\text { Prospective, } \\
\text { multicenter, } \\
\text { paired-cohort }\end{array}$ & $\begin{array}{c}\text { sensitivity 93\%, } \\
\text { specificity } 41 \%\end{array}$ & $\begin{array}{l}\text { Could avoid 27\% of biopsies } \\
\text { TRUS directed by MRI, could diagnose 18\% } \\
\text { more csPCas }\end{array}$ \\
\hline mpUS [68] & 1040 & $\begin{array}{c}\text { Prospective, } \\
\text { multicenter }\end{array}$ & $\begin{array}{c}\text { mpUS sensitivity 94\%, } \\
\text { specificity 22\% }\end{array}$ & $\begin{array}{l}\text { Results were similar with mpMRI sensitivity 93\%, } \\
\text { specificity 23\% }\end{array}$ \\
\hline 4Kscore, MRI, & 266 & $\begin{array}{c}\text { Retrospective, } \\
\text { unicentric }\end{array}$ & $\begin{array}{c}\text { 4Kscore and MRI if } \\
4 \mathrm{~K}>7.5\end{array}$ & $\begin{array}{l}\text { 4kscore followed by MRI if 4K }>7.5 \text { and biopsy if } \\
\text { MRI is positive } \\
\text { or } \\
\text { biopsy if MRI negative and 4K }>18\end{array}$ \\
\hline
\end{tabular}

From the imaging point of view, mpMRI has established its role in the diagnosis of PCa, and US represents a technique that is catching up, but these two are the only ones used in cancer diagnostics. Another technique that proved its usefulness is the positron emission tomography-computer tomography (PET-CT) for staging and metastasis identification. In PCa, the tracers used are Choline and Gallium 68 prostatic specific membrane antigen $\left({ }^{68} \mathrm{Ga}-\mathrm{PSMA}\right)$, the latter proving its superiority in identifying metabolically active metastasis outside the prostate, it identified metastasis in $48 \%$ of patients that had negative choline PET-CTs. It can identify lymph nodes as small as $8 \mathrm{~mm}$. The prostate or prostate bed (after radical prostatectomy) is difficult to evaluate because of the tracer accumulates in the urine, especially in the bladder and urethra $[77,78]$.

MicroRNAs (MIRNAs) represent small (21-25 nucleotides) non-coding RNAs that regulate gene and protein expression. They have tumor repressor or oncogenic roles, are tissue-specific, and can be found in blood, urine, or tissue. Their levels can be modified in all stages of PCa, from incipient tumors to metastatic states. These molecules look promising but further studies for validations are needed $[79,80]$.

Standard Risk Calculators such as ERSPC RPCRC, PCPT (Prostate Cancer Prevention Trial) model, and the Prostate Class model, are validated and can be used to stratify csPCa better. Novel ones like ERSPC RPCRC3 include new biomarkers (PHI) and showed better detection of PCa with an AUC of 0.75 [15].

In a scenario where ERSPC RPCRC is performed, if the result is positive, the MRI would have been performed, and this strategy could reduce the MRIs and prostate biopsies by $37 \%$ while missing about $4 \%$ of csPCa [81].

Another essential aspect that concerns physicians is economic burden. In the US, for men between 55-69, a low frequency (4 years) active surveillance could be cost-efficient, at a threshold of USD 10,000, but it loses its cost efficiency if there are shorter screening intervals or if there is a need for immediate treatment [24].

In the screening programs for Chinese men, adopting a PHI strategy proved to be more cost-effective than the PSA strategy [82].

MpMRI and biopsy could cost around GBP 965/patient, but using PHI $\geq 30$ before mpMRI, reduced costs with about 20\% (GBP 774/patient) [41].

Ultrasound scanners cost about GBP 10,000 [71], but they are cheaper in comparison with an MRI system. Another problem is the training of the specialists, but this is valid for both techniques.

In Australia, the biopsy process median costs were about 2711 Australian Dollars if the transrectal technique is conducted, and 3441 Australian Dollars for the transperineal approach. The introduction of mpMRI as a triage agent allowed them to save 784 Australian Dollars per patient and reduced the number of biopsies by $47 \%$ [27].

In the United States, Niranjan et al. developed a model in which patients with PSA $>3 \mathrm{ng} / \mathrm{mL}$ would undergo TRUSBx as part of the standard of care for diagnosing PCa or they would have performed one of three tests (PHI, 4Kscore, SelectMDx). The standard of care cost was about USD 3800 per patient using PHI or SelectMDx as part of the process, lowering the charges, but on the other hand, 4Kscore proved to be more expensive [83]. 
In Table 3 are represented the economic impact of the data mentioned above.

Table 3. Economic aspects of different tests around the world.

\begin{tabular}{ccc}
\hline Country & Test & Costs \\
\hline Germany [34] & PHID & 100 EUR \\
\hline China, Hong Kong [82] & PHI & 370 USD \\
\hline & PHI & 80 USD \\
\cline { 2 - 3 } & 4Kscore & 500 USD \\
\cline { 2 - 3 } & PCA 3 & 300 USD \\
\cline { 2 - 3 } & MiPS & 700 USD \\
\cline { 2 - 3 } & SelectMDx & 300 USD \\
\cline { 2 - 3 } & ERSPC RPCRC Risk Calculator & 0 \\
\cline { 2 - 3 } & PCPT Risk Calculator & 1000 USD \\
\hline Europe [15] & MRI & $300-500$ EUR \\
\hline \multirow{2}{*}{ UK [71] } & MRI & 35,000-150,000 USD \\
\cline { 2 - 3 } & UR Scanner & Approx. 3 million USD \\
\hline & MRI + biopsy & 965 GBP \\
\hline
\end{tabular}

\section{Conclusions}

The era in which TRUSbx was performed based only on PSA values is gone. PSA is slowly becoming an indicator for further investigations, not a test to determine if a patient should proceed to biopsy or not.

PHI, 4Kscore, STHLM3, MRI and mpUS showed superior sensitivity and specificity over PSA in the diagnosing process, active surveillance, discrimination between malign and benign, and between indolent and aggressive tumors. In addition, recently merged tests proved superiority over singular ones, and they can provide a smooth patient flow that will not overwhelm the system.

These tests were investigated for a short period of time; prospective studies are needed to determine if they play a role in survival, metastatic advancement, or cancer-specific mortality. To date, there is no test available that is accurate enough to skip tissue sampling. The validation of these biomarkers needs to be confirmed on large cohorts because statistical difference does not necessarily mean clinical difference. In summary, it is evident that biomarkers come with a benefit in the decision making of prostate cancer diagnosis, and moreover, most of them are easily accessible.

Finally, in the future, biomarkers and imaging techniques for prostate cancer diagnosis will have to be used in an interconnected routine rather than in a competitive one, in order to determine the best patient selection.

Author Contributions: Conceptualization V.C.M., R.A.M., I.B.N., B.P., I.C.; methodology V.C.M., R.A.M., V.H.S.; writing-Original draft, V.C.M., R.A.M., D.G.; writing-Review and editing, V.C.M., R.A.M.; visualization, P.A.C., V.H.S.; supervision, I.B.N., B.P., I.C., P.A.C.; project administration, V.C.M., I.C.; funding acquisition, V.C.M. All authors have read and agreed to the published version of the manuscript.

Funding: This research was supported by the research grant "MicroRNAs in prostate adenocarcinoma. Diagnostic, prognostic and therapeutic role"-PCD1530/43 founded by "Iuliu Hatieganu" University of Medicine and Pharmacy, Cluj-Napoca, Romania.

Conflicts of Interest: The authors declare no conflict of interest. The funders had no role in the design of the study; in the collection, analyses, or interpretation of data; in the writing of the manuscript, or in the decision to publish the results. 
Abbreviations

$4 \mathrm{~K}$

${ }^{68}$ Ga-PET-CT

ACS

ADC

AS

AUA

AUC

BMI

BPMRI

BPH

BRCA

CAP

CDUS

CEUS

csPCa

DCE

DRE

DWI

EAU

ERSPC

ESMO

FDA

fPSA

GG

HG

ISUP

MIC1

MicroRNAs

Mp MRI

Mp US

MRGB

MSMB

NPV

PCa

PCA3

PCPT

PET-CT

PHID

PHI

PI-RADS

PLCO

PPV

PRI-MUS

PSA

PSAD

ROC

RPCRC

SB

SE

SMI

STHLM3 (S3M)

SWE

T2W
4 kalikrein score

Gallium 68 positron emission tomography- computer tomography American Cancer Society

apparent diffusion coefficient

active surveillance

American Urology Association

area under the curve

body mass index

bi parametric magnetic resonance imaging

benign prostatic hyperplasia

breast cancer gene

The Cluster Randomized Trial of PSA Testing for Prostate Cancer

color Doppler ultrasound

contrast-enhanced ultrasound

clinically significant $\mathrm{PCa}$

dynamic contrast enhancement

digital rectal examination

diffusion-weighted imaging

European Association of Urology

European Randomized Study of Screening for Prostate Cancer trial

European Society of Medical Oncology

Food and Drug Administration

freePSA

Gleason Grade

high grade

International Society of Urological Pathology

macrophage inhibitory cytokine-1

MiRNA

multiparametric magnetic resonance imaging

multiparametric ultrasound

magnetic resonance guided biopsy

microseminoprotein beta

negative predictive value

prostate cancer

prostate cancer antigen 3

Prostate Cancer Prevention Trial

positron emission tomography- computer tomography

prostate health index density

prostate health index

Prostate Imaging-Reporting and Data System

The Prostate, Lung, Colorectal and Ovarian

positive predictive value

Prostate risk identification-micro ultrasound

prostatic specific antigen

PSA density

receiver operating characteristics

Rotterdam Prostate Cancer Risk Calculator

systematic biopsy

strain elastography

spectacular micro-vascular imaging

Stockholm 3 test

shear wave elastography

$\mathrm{T} 2$ weighted image 
TB

TP

TPM

tPSA

TR

TRES

TRUS

TRUSGB

\author{
targeted biopsy \\ transperineal \\ template prostate mapping \\ Total PSA \\ transrectal \\ transrectal elastography \\ TransRectal UltraSound \\ transrectal ultrasound-guided biopsy
}

\section{References}

1. Nassir, A.M. A Piece in Prostate Cancer Puzzle: Future Perspective of Novel Molecular Signatures. Saudi J. Biol. Sci. 2020, 27, 1148-1154. [CrossRef]

2. Barsouk, A.; Padala, S.A.; Vakiti, A.; Mohammed, A.; Saginala, K.; Thandra, K.C.; Rawla, P.; Barsouk, A. Epidemiology, Staging and Management of Prostate Cancer. Med. Sci. 2020, 8, 28. [CrossRef]

3. Pilleron, S.; Soto-Perez-de-Celis, E.; Vignat, J.; Ferlay, J.; Soerjomataram, I.; Bray, F.; Sarfati, D. Estimated Global Cancer Incidence in the Oldest Adults in 2018 and Projections to 2050. Int. J. Cancer 2020, ijc.33232. [CrossRef] [PubMed]

4. Bray, F.; Ferlay, J.; Soerjomataram, I.; Siegel, R.L.; Torre, L.A.; Jemal, A. Global Cancer Statistics 2018: GLOBOCAN Estimates of Incidence and Mortality Worldwide for 36 Cancers in 185 Countries. CA. Cancer J. Clin. 2018, 68, 394-424. [CrossRef] [PubMed]

5. Merriel, S.W.D.; Funston, G.; Hamilton, W. Prostate Cancer in Primary Care. Adv. Ther. 2018, 35, $1285-1294$. [CrossRef] [PubMed]

6. Mottet, N.; van den Bergh, R.C.N.; Briers, E.; Cornford, P.; de Santis, M.; Fanti, S.; Gillessen, S.; Grummet, J.; Henry, A.M.; Lam, T.B.; et al. EAU-EANM-ESTRO-ESUR-SIOG Guidelines on Prostate Cancer. Eur. Assoc. Urol. 2019, 75, 889-890.

7. McDougal, W.S.; Wein, J.A.; Kavoussi, R.L.; Partin, W.A.; Peters, C. Prostate Cancer Tumor Markers. In Campbell-Walsh Urology; Elsevier: Philadelphia, PA, USA, 2016; pp. 2568-2569.

8. Mottet, N.; Cornford, P.; van den Bergh, R.C.N.; Briers, E.; De Santis, M.; Fanti, S.; Gillessen, S.; Grummet, J.; Henry, A.M.; Lam, T.B.; et al. EAU Guidelines. Edn. presented at the EAU Annual Congress Amsterdam; EAU Guidelines Office: Arnhem, The Netherlands, 2020.

9. Thomsen, F.B.; Brasso, K.; Klotz, L.H.; Røder, M.A.; Berg, K.D.; Iversen, P. Active Surveillance for Clinically Localized Prostate Cancer-A Systematic Review. J. Surg. Oncol. 2014, 109, 830-835. [CrossRef]

10. Heidenreich, A.; Bastian, P.J.; Bellmunt, J.; Bolla, M.; Joniau, S.; van der Kwast, T.; Mason, M.; Matveev, V.; Wiegel, T.; Zattoni, F.; et al. EAU Guidelines on Prostate Cancer. Part 1: Screening, Diagnosis, and Local Treatment with Curative Intent-Update 2013. Eur. Urol. 2014, 65, 124-137. [CrossRef]

11. Wilt, T.J.; Brawer, M.K.; Jones, K.M.; Barry, M.J.; Aronson, W.J.; Fox, S.; Gingrich, J.R.; Wei, J.T.; Gilhooly, P.; Grob, B.M.; et al. Radical Prostatectomy versus Observation for Localized Prostate Cancer. N. Engl. J. Med. 2012, 367, 203-213. [CrossRef]

12. Hamdy, F.C.; Donovan, J.L.; Lane, J.A.; Mason, M.; Metcalfe, C.; Holding, P.; Wade, J.; Noble, S.; Garfield, K.; Young, G.; et al. Active Monitoring, Radical Prostatectomy and Radical Radiotherapy in PSA-Detected Clinically Localised Prostate Cancer: The ProtecT Three-Arm RCT. Health Technol. Assess. 2020, 24, 1-176. [CrossRef]

13. Houédé, N.; Rébillard, X.; Bouvet, S.; Kabani, S.; Fabbro-Peray, P.; Trétarre, B.; Ménégaux, F. Impact on Quality of Life 3 Years after Diagnosis of Prostate Cancer Patients below 75 at Diagnosis: An Observational Case-Control Study. BMC Cancer 2020, 20, 757. [CrossRef] [PubMed]

14. Tan, G.H.; Nason, G.; Ajib, K.; Woon, D.T.S.; Herrera-Caceres, J.; Alhunaidi, O.; Perlis, N. Smarter Screening for Prostate Cancer. World J. Urol. 2019, 37, 991-999. [CrossRef] [PubMed]

15. Osses, D.; Roobol, M.; Schoots, I. Prediction Medicine: Biomarkers, Risk Calculators and Magnetic Resonance Imaging as Risk Stratification Tools in Prostate Cancer Diagnosis. Int. J. Mol. Sci. 2019, 20, 1637. [CrossRef]

16. Schoots, I.G.; Roobol, M.J. Multivariate Risk Prediction Tools Including MRI for Individualized Biopsy Decision in Prostate Cancer Diagnosis: Current Status and Future Directions. World J. Urol. 2020, 38, 517-529. [CrossRef] [PubMed] 
17. Gomez Gomez, E.; Salamanca Bustos, J.J.; Carrasco Valiente, J.; Fernandez Rueda, J.L.; Blanca, A.; Valero Rosa, J.; Bravo Arrebola, I.; Marquez López, J.; Jimenez Vacas, J.M.; Luque, R.; et al. Observational Study Comparing the Accuracy/Variability between the ERSPC and the PCPT Risk Calculators for the Prediction of Significant Prostate Cancer in Patients with PSA $<10$ ng/mL. BMJ open 2019, 9, e031032. [CrossRef]

18. Naji, L.; Randhawa, H.; Sohani, Z.; Dennis, B.; Lautenbach, D.; Kavanagh, O.; Bawor, M.; Banfield, L.; Profetto, J. Digital Rectal Examination for Prostate Cancer Screening in Primary Care: A Systematic Review and Meta-Analysis. Ann. Fam. Med. 2018, 16, 149-154. [CrossRef]

19. Herrera-Caceres, J.O.; Wettstein, M.S.; Goldberg, H.; Toi, A.; Chandrasekar, T.; Woon, D.T.S.; Ahmad, A.E.; Sanmamed-Salgado, N.; Alhunaidi, O.; Ajib, K.; et al. Utility of Digital Rectal Examination in a Population with Prostate Cancer Treated with Active Surveillance. Can. Urol. Assoc. J. 2020, 14. [CrossRef]

20. Gosselaar, C.; Roobol, M.J.; Roemeling, S.; Schröder, F.H. The Role of the Digital Rectal Examination in Subsequent Screening Visits in the European Randomized Study of Screening for Prostate Cancer (ERSPC), Rotterdam. Eur. Urol. 2008, 54, 581-588. [CrossRef]

21. Eckersberger, E.; Finkelstein, J.; Sadri, H.; Margreiter, M.; Taneja, S.S.; Lepor, H.; Djavan, B. Screening for Prostate Cancer: A Review of the ERSPC and PLCO Trials. Rev. Urol. 2009, 11, 127-133.

22. Gupta, A.; Roobol, M.J.; Savage, C.J.; Peltola, M.; Pettersson, K.; Scardino, P.T.; Vickers, A.J.; Schröder, F.H.; Lilja, H. A Four-Kallikrein Panel for the Prediction of Repeat Prostate Biopsy: Data from the European Randomized Study of Prostate Cancer Screening in Rotterdam, Netherlands. Br. J. Cancer 2010, 103, 708-714. [CrossRef]

23. Vickers, A.J.; Cronin, A.M.; Roobol, M.J.; Savage, C.J.; Peltola, M.; Pettersson, K.; Scardino, P.T.; Schroder, F.H.; Lilja, H. A Four-Kallikrein Panel Predicts Prostate Cancer in Men with Recent Screening: Data from the European Randomized Study of Screening for Prostate Cancer, Rotterdam. Clin. Cancer Res. 2010, 16, 3232-3239. [CrossRef] [PubMed]

24. Tikkinen, K.A.O.; Dahm, P.; Lytvyn, L.; Heen, A.F.; Vernooij, R.W.M.; Siemieniuk, R.A.C.; Wheeler, R.; Vaughan, B.; Fobuzi, A.C.; Blanker, M.H.; et al. Prostate Cancer Screening with Prostate-Specific Antigen (PSA) Test: A Clinical Practice Guideline. BMJ 2018, k3581. [CrossRef] [PubMed]

25. Schröder, F.H.; Hugosson, J.; Roobol, M.J.; Tammela, T.L.J.; Zappa, M.; Nelen, V.; Kwiatkowski, M.; Lujan, M.; Määttänen, L.; Lilja, H.; et al. Screening and Prostate Cancer Mortality: Results of the European Randomised Study of Screening for Prostate Cancer (ERSPC) at 13 Years of Follow-Up. Lancet 2014, 384, 2027-2035. [CrossRef]

26. Tohi, Y.; Kato, T.; Matsumoto, R.; Shinohara, N.; Shiga, K.; Yokomizo, A.; Nakamura, M.; Kume, H.; Mitsuzuka, K.; Sasaki, H.; et al. The Impact of Complications after Initial Prostate Biopsy on Repeat Protocol Biopsy Acceptance Rate. Results from the Prostate Cancer Research International: Active Surveillance JAPAN Study. Int. J. Clin. Oncol. 2020. [CrossRef] [PubMed]

27. Roberts, M.J.; Macdonald, A.; Ranasinghe, S.; Bennett, H.; Teloken, P.E.; Harris, P.; Paterson, D.; Coughlin, G.; Dunglison, N.; Esler, R.; et al. Transrectal versus Transperineal Prostate Biopsy under Intravenous Anaesthesia: A Clinical, Microbiological and Cost Analysis of 2048 Cases over 11 Years at a Tertiary Institution. Prostate Cancer Prostatic Dis. 2020. [CrossRef] [PubMed]

28. Moradi, A.; Srinivasan, S.; Clements, J.; Batra, J. Beyond the Biomarker Role: Prostate-Specific Antigen (PSA) in the Prostate Cancer Microenvironment. Cancer Metastasis Rev. 2019, 38, 333-346. [CrossRef]

29. Perera, M.; Mirchandani, R.; Papa, N.; Breemer, G.; Effeindzourou, A.; Smith, L.; Swindle, P.; Smith, E. PSA-Based Machine Learning Model Improves Prostate Cancer Risk Stratification in a Screening Population. World J. Urol. 2020. [CrossRef]

30. Birkeland, S.; Pedersen, S.S.; Haakonsson, A.K.; Barry, M.J.; Rottmann, N. Men's View on Participation in Decisions about Prostate-Specific Antigen (PSA) Screening: Patient and Public Involvement in Development of a Survey. BMC Med. Inform. Decis. Mak. 2020, 20, 65. [CrossRef]

31. Parker, C.; Castro, E.; Fizazi, K.; Heidenreich, A.; Ost, P.; Procopio, G.; Tombal, B.; Gillessen, S. Prostate Cancer: ESMO Clinical Practice Guidelines for Diagnosis, Treatment and Follow-Up. Ann. Oncol. 2020, 31, 1119-1134. [CrossRef]

32. Smith, R.A.; Andrews, K.S.; Brooks, D.; Fedewa, S.A.; Manassaram-Baptiste, D.; Saslow, D.; Wender, R.C. Cancer Screening in the United States, 2019: A Review of Current American Cancer Society Guidelines and Current Issues in Cancer Screening. CA. Cancer J. Clin. 2019, 69, 184-210. [CrossRef] 
33. Loeb, S.; Bruinsma, S.M.; Nicholson, J.; Briganti, A.; Pickles, T.; Kakehi, Y.; Carlsson, S.V.; Roobol, M.J. Active Surveillance for Prostate Cancer: A Systematic Review of Clinicopathologic Variables and Biomarkers for Risk Stratification. Eur. Urol. 2015, 67, 619-626. [CrossRef] [PubMed]

34. Schulze, A.; Christoph, F.; Sachs, M.; Schroeder, J.; Stephan, C.; Schostak, M.; Koenig, F. Use of the Prostate Health Index and Density in 3 Outpatient Centers to Avoid Unnecessary Prostate Biopsies. Urol. Int. 2020, 104, 181-186. [CrossRef] [PubMed]

35. Takeshima, Y.; Tanaka, Y.; Takemura, K.; Nakazono, S.; Yamashita, E.; Kume, H. Evaluating the Efficacy of a Low-Cost Cognitive MRI-Targeted Prostate Biopsy Protocol: Is There Still a Role for Lower Volume Centers in the Prostate Imaging Reporting and Data System (PI-RADS) Version 2 Era? Int. Urol. Nephrol. 2020. [CrossRef] [PubMed]

36. Nordström, T.; Akre, O.; Aly, M.; Grönberg, H.; Eklund, M. Prostate-Specific Antigen (PSA) Density in the Diagnostic Algorithm of Prostate Cancer. Prostate Cancer Prostatic Dis. 2018, 21, 57-63. [CrossRef] [PubMed]

37. Vickers, A.; Carlsson, S.V.; Cooperberg, M. Routine Use of Magnetic Resonance Imaging for Early Detection of Prostate Cancer Is Not Justified by the Clinical Trial Evidence. Eur. Urol. 2020. [CrossRef]

38. Jin, W.; Fei, X.; Wang, X.; Song, Y.; Chen, F. Detection and Prognosis of Prostate Cancer Using Blood-Based Biomarkers. Mediat. Inflamm. 2020, 2020, 1-11. [CrossRef]

39. Brönimann, S.; Pradere, B.; Karakiewicz, P.; Abufaraj, M.; Briganti, A.; Shariat, S.F. An Overview of Current and Emerging Diagnostic, Staging and Prognostic Markers for Prostate Cancer. Expert Rev. Mol. Diagn. 2020, 1-10. [CrossRef]

40. Heijnsdijk, E.A.M.; Denham, D.; de Koning, H.J. The Cost-Effectiveness of Prostate Cancer Detection with the Use of Prostate Health Index. Value Health 2016, 19, 153-157. [CrossRef]

41. Kim, L.; Boxall, N.; George, A.; Burling, K.; Acher, P.; Aning, J.; McCracken, S.; Page, T.; Gnanapragasam, V.J. Clinical Utility and Cost Modelling of the Phi Test to Triage Referrals into Image-Based Diagnostic Services for Suspected Prostate Cancer: The PRIM (Phi to RefIne Mri) Study. BMC Med. 2020, 18, 95. [CrossRef]

42. Schwen, Z.R.; Mamawala, M.; Tosoian, J.J.; Druskin, S.C.; Ross, A.E.; Sokoll, L.J.; Epstein, J.I.; Carter, H.B.; Gorin, M.A.; Pavlovich, C.P. Prostate Health Index and Multiparametric Magnetic Resonance Imaging to Predict Prostate Cancer Grade Reclassification in Active Surveillance. BJU Int. 2020. [CrossRef]

43. Nassir, A.M.; Kamel, H.F.M. Explication of the Roles of Prostate Health Index (PHI) and Urokinase Plasminogen Activator (UPA) as Diagnostic and Predictor Tools for Prostate Cancer in Equivocal PSA Range of 4-10 Ng/ML. Saudi J. Biol. Sci. 2020, 27, 1975-1984. [CrossRef] [PubMed]

44. Lin, D.W.; Newcomb, L.F.; Brown, M.D.; Sjoberg, D.D.; Dong, Y.; Brooks, J.D.; Carroll, P.R.; Cooperberg, M.; Dash, A.; Ellis, W.J.; et al. Evaluating the Four Kallikrein Panel of the 4Kscore for Prediction of High-Grade Prostate Cancer in Men in the Canary Prostate Active Surveillance Study. Eur. Urol. 2017, 72, 448-454. [CrossRef] [PubMed]

45. Braun, K.; Sjoberg, D.D.; Vickers, A.J.; Lilja, H.; Bjartell, A.S. A Four-Kallikrein Panel Predicts High-Grade Cancer on Biopsy: Independent Validation in a Community Cohort. Eur. Urol. 2016, 69, 505-511. [CrossRef] [PubMed]

46. Darst, B.F.; Chou, A.; Wan, P.; Pooler, L.; Sheng, X.; Vertosick, E.A.; Conti, D.V.; Wilkens, L.R.; Le Marchand, L.; Vickers, A.J.; et al. The Four-Kallikrein Panel Is Effective in Identifying Aggressive Prostate Cancer in a Multiethnic Population. Cancer Epidemiol. Biomark. Prev. 2020, 29, 1381-1388. [CrossRef]

47. Verbeek, J.F.M.; Bangma, C.H.; Kweldam, C.F.; van der Kwast, T.H.; Kümmerlin, I.P.; van Leenders, G.J.L.H.; Roobol, M.J. Reducing Unnecessary Biopsies While Detecting Clinically Significant Prostate Cancer Including Cribriform Growth with the ERSPC Rotterdam Risk Calculator and 4Kscore. Urol. Oncol. Semin. Orig. Investig. 2019, 37, 138-144. [CrossRef]

48. Vigneswaran, H.T.; Discacciati, A.; Gann, P.H.; Grönberg, H.; Eklund, M.; Abern, M.R. Ethnic Variation in Prostate Cancer Detection: A Feasibility Study for Use of the Stockholm3 Test in a Multiethnic U.S. Cohort. Prostate Cancer Prostatic Dis. 2020. [CrossRef]

49. Grönberg, H.; Adolfsson, J.; Aly, M.; Nordström, T.; Wiklund, P.; Brandberg, Y.; Thompson, J.; Wiklund, F.; Lindberg, J.; Clements, M.; et al. Prostate Cancer Screening in Men Aged 50-69 Years (STHLM3): A Prospective Population-Based Diagnostic Study. Lancet Oncol. 2015, 16, 1667-1676. [CrossRef]

50. Ström, P.; Nordström, T.; Aly, M.; Egevad, L.; Grönberg, H.; Eklund, M. The Stockholm-3 Model for Prostate Cancer Detection: Algorithm Update, Biomarker Contribution, and Reflex Test Potential. Eur. Urol. 2018, 74, 204-210. [CrossRef] 
51. Grönberg, H.; Eklund, M.; Picker, W.; Aly, M.; Jäderling, F.; Adolfsson, J.; Landquist, M.; Haug, E.S.; Ström, P.; Carlsson, S.; et al. Prostate Cancer Diagnostics Using a Combination of the Stockholm3 Blood Test and Multiparametric Magnetic Resonance Imaging. Eur. Urol. 2018, 74, 722-728. [CrossRef]

52. Palsdottir, T.; Nordström, T.; Aly, M.; Jäderling, F.; Clements, M.; Grönberg, H.; Eklund, M. A Unified Prostate Cancer Risk Prediction Model Combining the Stockholm3 Test and Magnetic Resonance Imaging. Eur. Urol. Oncol. 2019, 2, 490-496. [CrossRef]

53. Thestrup, K.C.D.; Logager, V.; Baslev, I.; Møller, J.M.; Hansen, R.H.; Thomsen, H.S. Biparametric versus Multiparametric MRI in the Diagnosis of Prostate Cancer. Acta Radiol. Open 2016, 5, 205846011666304. [CrossRef] [PubMed]

54. Kasivisvanathan, V.; Rannikko, A.S.; Borghi, M.; Panebianco, V.; Mynderse, L.A.; Vaarala, M.H.; Briganti, A.; Budäus, L.; Hellawell, G.; Hindley, R.G.; et al. MRI-Targeted or Standard Biopsy for Prostate-Cancer Diagnosis. N. Engl. J. Med. 2018, 378, 1767-1777. [CrossRef] [PubMed]

55. Labra, A.; González, F.; Silva, C.; Franz, G.; Pinochet, R.; Gupta, R.T. MRI/TRUS Fusion vs. Systematic Biopsy: Intra-Patient Comparison of Diagnostic Accuracy for Prostate Cancer Using PI-RADS V2. Abdom. Radiol. 2020, 45, 2235-2243. [CrossRef]

56. Noh, T.I.; Tae, J.H.; Kim, H.K.; Shim, J.S.; Kang, S.G.; Sung, D.J.; Cheon, J.; Lee, J.G.; Kang, S.H. Diagnostic Accuracy and Value of Magnetic Resonance Imaging-Ultrasound Fusion Transperineal Targeted and Template Systematic Prostate Biopsy Based on Bi-Parametric Magnetic Resonance Imaging. Cancer Res. Treat. 2020, 52, 714-721. [CrossRef] [PubMed]

57. Marzouk, K.; Ehdaie, B.; Vertosick, E.; Zappala, S.; Vickers, A. Developing an Effective Strategy to Improve the Detection of Significant Prostate Cancer by Combining the 4Kscore and Multiparametric MRI. Urol. Oncol. Semin. Orig. Investig. 2019, 37, 672-677. [CrossRef] [PubMed]

58. Klotz, L.; Pond, G.; Loblaw, A.; Sugar, L.; Moussa, M.; Berman, D.; Van der Kwast, T.; Vesprini, D.; Milot, L.; Kebabdjian, M.; et al. Randomized Study of Systematic Biopsy Versus Magnetic Resonance Imaging and Targeted and Systematic Biopsy in Men on Active Surveillance (ASIST): 2-Year Postbiopsy Follow-Up. Eur. Urol. 2020, 77, 311-317. [CrossRef]

59. Drudi, F.M.; Cantisani, V.; Angelini, F.; Ciccariello, M.; Messineo, D.; Ettorre, E.; Liberatore, M.; Scialpi, M. Multiparametric MRI Versus Multiparametric US in the Detection of Prostate Cancer. Anticancer Res. 2019, 39, 3101-3110. [CrossRef]

60. Zhen, L.; Liu, X.; Yegang, C.; Yongjiao, Y.; Yawei, X.; Jiaqi, K.; Xianhao, W.; Yuxuan, S.; Rui, H.; Wei, Z.; et al. Accuracy of Multiparametric Magnetic Resonance Imaging for Diagnosing Prostate Cancer: A Systematic Review and Meta-Analysis. BMC Cancer 2019, 19, 1244. [CrossRef]

61. Morote, J.; Celma, A.; Roche, S.; de Torres, I.M.; Mast, R.; Semedey, M.E.; Regis, L.; Planas, J. Who Benefits from Multiparametric Magnetic Resonance Imaging After Suspicion of Prostate Cancer? Eur. Urol. Oncol. 2019, 2, 664-669. [CrossRef]

62. Porpiglia, F.; Russo, F.; Manfredi, M.; Mele, F.; Fiori, C.; Bollito, E.; Papotti, M.; Molineris, I.; Passera, R.; Regge, D. The Roles of Multiparametric Magnetic Resonance Imaging, PCA3 and Prostate Health Index-Which Is the Best Predictor of Prostate Cancer after a Negative Biopsy? J. Urol. 2014, 192, 60-66. [CrossRef]

63. van der Leest, M.; Cornel, E.; Israël, B.; Hendriks, R.; Padhani, A.R.; Hoogenboom, M.; Zamecnik, P.; Bakker, D.; Setiasti, A.Y.; Veltman, J.; et al. Head-to-Head Comparison of Transrectal Ultrasound-Guided Prostate Biopsy Versus Multiparametric Prostate Resonance Imaging with Subsequent Magnetic Resonance-Guided Biopsy in Biopsy-Naïve Men with Elevated Prostate-Specific Antigen: A Large Prospective Mu. Eur. Urol. 2019, 75, 570-578. [CrossRef]

64. Correas, J.-M.; Halpern, E.J.; Barr, R.G.; Ghai, S.; Walz, J.; Bodard, S.; Dariane, C.; de la Rosette, J. Advanced Ultrasound in the Diagnosis of Prostate Cancer. World J. Urol. 2020. [CrossRef] [PubMed]

65. Yunkai, Z.; Yaqing, C.; Jun, J.; Tingyue, Q.; Weiyong, L.; Yuehong, Q.; Wenbin, G.; Lifeng, W.; Jun, Q. Comparison of Contrast-Enhanced Ultrasound Targeted Biopsy versus Standard Systematic Biopsy for Clinically Significant Prostate Cancer Detection: Results of a Prospective Cohort Study with 1024 Patients. World J. Urol. 2019, 37, 805-811. [CrossRef] [PubMed]

66. Ghai, S.; Eure, G.; Fradet, V.; Hyndman, M.E.; McGrath, T.; Wodlinger, B.; Pavlovich, C.P. Assessing Cancer Risk on Novel $29 \mathrm{MHz}$ Micro-Ultrasound Images of the Prostate: Creation of the Micro-Ultrasound Protocol for Prostate Risk Identification. J. Urol. 2016, 196, 562-569. [CrossRef] 
67. Abouassaly, R.; Klein, E.A.; El-Shefai, A.; Stephenson, A. Impact of Using 29 MHz High-Resolution Micro-Ultrasound in Real-Time Targeting of Transrectal Prostate Biopsies: Initial Experience. World J. Urol. 2020, 38, 1201-1206. [CrossRef] [PubMed]

68. Klotz, L.; Lughezzani, G.; Maffei, D.; Sanchez, A.; Pereira, J.G.; Staerman, F.; Cash, H.; Luger, F.; Lopez, L.; Sanchez-Salas, R.; et al. Comparison of Micro-Ultrasound and Multiparametric Magnetic Resonance Imaging for Prostate Cancer: A Multicenter, Prospective Analysis. Can. Urol. Assoc. J. 2020, 15. [CrossRef]

69. Zhu, Y.-C.; Shan, J.; Zhang, Y.; Jiang, Q.; Wang, Y.-B.; Deng, S.-H.; Qu, Q.-H.; Li, Q. Prostate Cancer Vascularity: Superb Microvascular Imaging Ultrasonography with Histopathology Correlation. Med. Sci. Monit. 2019, 25, 8571-8578. [CrossRef]

70. Lughezzani, G.; Saita, A.; Lazzeri, M.; Paciotti, M.; Maffei, D.; Lista, G.; Hurle, R.; Buffi, N.M.; Guazzoni, G.; Casale, P. Comparison of the Diagnostic Accuracy of Micro-Ultrasound and Magnetic Resonance Imaging/Ultrasound Fusion Targeted Biopsies for the Diagnosis of Clinically Significant Prostate Cancer. Eur. Urol. Oncol. 2019, 2, 329-332. [CrossRef]

71. Grey, A.D.R.; Connor, M.J.; Tam, J.; Loch, T. Can Transrectal Prostate Ultrasound Compete with Multiparametric MRI in the Detection of Clinically Significant Prostate Cancer? Transl. Androl. Urol. 2020, 9, 1492-1500. [CrossRef]

72. Falagario, U.G.; Martini, A.; Wajswol, E.; Treacy, P.-J.; Ratnani, P.; Jambor, I.; Anastos, H.; Lewis, S.; Haines, K.; Cormio, L.; et al. Avoiding Unnecessary Magnetic Resonance Imaging (MRI) and Biopsies: Negative and Positive Predictive Value of MRI According to Prostate-Specific Antigen Density, 4Kscore and Risk Calculators. Eur. Urol. Oncol. 2019. [CrossRef]

73. Wysock, J.S.; Becher, E.; Persily, J.; Loeb, S.; Lepor, H. Concordance and Performance of 4 Kscore and SelectMDx for Informing Decision to Perform Prostate Biopsy and Detection of Prostate Cancer. Urology 2020, 141, 119-124. [CrossRef]

74. Wiemer, L.; Hollenbach, M.; Heckmann, R.; Kittner, B.; Plage, H.; Reimann, M.; Asbach, P.; Friedersdorff, F.; Schlomm, T.; Hofbauer, S.; et al. Evolution of Targeted Prostate Biopsy by Adding Micro-Ultrasound to the Magnetic Resonance Imaging Pathway. Eur. Urol. Focus 2020. [CrossRef] [PubMed]

75. Gadzinski, A.J.; Cooperberg, M.R. Prostate Cancer Markers. U.S. Patent 6,673,545, 6 January 2018. [CrossRef]

76. Ahmed, H.U.; El-Shater Bosaily, A.; Brown, L.C.; Gabe, R.; Kaplan, R.; Parmar, M.K.; Collaco-Moraes, Y.; Ward, K.; Hindley, R.G.; Freeman, A.; et al. Diagnostic Accuracy of Multi-Parametric MRI and TRUS Biopsy in Prostate Cancer (PROMIS): A Paired Validating Confirmatory Study. Lancet 2017, 389, 815-822. [CrossRef]

77. Afaq, A.; Bomanji, J. Prostate-Specific Membrane Antigen Positron Emission Tomography in the Management of Recurrent Prostate Cancer. Br. Med. Bull. 2018, 128, 37-48. [CrossRef] [PubMed]

78. Oh, S.W.; Cheon, G.J. Prostate-Specific Membrane Antigen PET Imaging in Prostate Cancer: Opportunities and Challenges. Korean J. Radiol. 2018, 19, 819. [CrossRef] [PubMed]

79. Munteanu, V.C.; Munteanu, R.A.; Onaciu, A.; Berindan-Neagoe, I.; Petrut, B.; Coman, I. MiRNA-Based Inspired Approach in Diagnosis of Prostate Cancer. Medicina 2020, 56, 94. [CrossRef]

80. Fredsøe, J.; Rasmussen, A.K.I.; Mouritzen, P.; Bjerre, M.T.; Østergren, P.; Fode, M.; Borre, M.; Sørensen, K.D. Profiling of Circulating MicroRNAs in Prostate Cancer Reveals Diagnostic Biomarker Potential. Diagnostics 2020, 10, 188. [CrossRef]

81. Mannaerts, C.K.; Gayet, M.; Verbeek, J.F.; Engelbrecht, M.R.W.; Savci-Heijink, C.D.; Jager, G.J.; Gielens, M.P.M.; van der Linden, H.; Beerlage, H.P.; de Reijke, T.M.; et al. Prostate Cancer Risk Assessment in Biopsy-Naïve Patients: The Rotterdam Prostate Cancer Risk Calculator in Multiparametric Magnetic Resonance Imaging-Transrectal Ultrasound (TRUS) Fusion Biopsy and Systematic TRUS Biopsy. Eur. Urol. Oncol. 2018, 1, 109-117. [CrossRef]

82. Teoh, J.Y.-C.; Leung, C.-H.; Wang, M.H.; Chiu, P.K.-F.; Yee, C.-H.; Ng, C.-F.; Wong, M.C.-S. The Cost-Effectiveness of Prostate Health Index for Prostate Cancer Detection in Chinese Men. Prostate Cancer Prostatic Dis. 2020. [CrossRef]

83. Sathianathen, N.J.; Kuntz, K.M.; Alarid-Escudero, F.; Lawrentschuk, N.L.; Bolton, D.M.; Murphy, D.G.; Weight, C.J.; Konety, B.R. Incorporating Biomarkers into the Primary Prostate Biopsy Setting: A Cost-Effectiveness Analysis. J. Urol. 2018, 200, 1215-1220. [CrossRef]

(C) 2020 by the authors. Licensee MDPI, Basel, Switzerland. This article is an open access article distributed under the terms and conditions of the Creative Commons Attribution (CC BY) license (http://creativecommons.org/licenses/by/4.0/). 